\title{
Studies of Charge Exchange in a High-Pressure Pulsed Electron Impact Source
}

\author{
D. K. Sen Sharma, P. M. Hierd, ${ }^{*}$ and J. L. Frankitn \\ Department of Chemistry, Rice University, Houston, Texas 77001
}

(Received 7 September 1971)

\begin{abstract}
A high pressure pulsed ion source has been used in a time-of-flight mass spectrometer in order to study the charge exchange reactions in $\mathrm{Ar}-\mathrm{H}_{2}$ and $\mathrm{Ar}-\mathrm{D}_{2}$ systems using the ion source in the Cermák mode of operation. As the source was used in a pulsed mode, it was possible to identify the various secondary ions arising from the charge exchange reactions in these systems. Very good agreement has been shown to exist between the experimental results and simple theoretical deductions. Calculations have been made to determine the cross sections for the charge exchange reactions between the various species from the experimental data.
\end{abstract}

\section{INTRODUCTION}

During the course of an investigation of ion-molecule reactions in this laboratory, the existence of the Cermák ${ }^{1}$ effect in the time-of-flight mass spectrometer was conclusively proved and suggestion was made $e^{2,3}$ about the possibility of utilizing this effect as a tool for further investigations. Two simple systems, $\mathrm{Ar}-\mathrm{H}_{2}$ and $\mathrm{Ar}-\mathrm{D}_{2}$, were selected for this purpose. Although the reaction of argon ions with the hydrogen and deuterium molecules and vice versa has been the subject of several investigations, ${ }^{4-15}$ we selected these two systems for our studies for two main reasons. First, argon is virtually monoisotopic, having $99.6 \%$ natural abundance for the isotope ${ }^{40} \mathrm{Ar}$ of greatest mass, so that low abundance isotopes did not produce any complicating background effects in the secondary spectrum. Second, the mass difference between argon and hydrogen, as well as between argon and deuterium, is large. This helped in separating and identifying ${ }^{3}$ the secondary ion peaks of different masses and from different sources unambiguously. The secondary spectrum consisted mainly of four peaks in each of the two systems studied, and this simple system permitted ready identification of the various peaks. The four peaks were $\mathrm{H}_{2}{ }^{+}$and $\mathrm{Ar}^{+}$secondary ions caused by charge exchange from both $\mathrm{H}_{2}^{+}$and $\mathrm{Ar}^{+}$primary ions. This investigation differs from that of other workers in that a high pressure pulsed ion source was used in the Cermák mode of operation. A pressure as high as $40 \mu$ was reached in the ion source.

Without incorporating any further instrumental modifications into the existing apparatus, we have been able to determine charge exchange cross sections which compare favorably with those obtained by other workers using much more elaborate experimental methods and apparatus.

\section{EXPERIMENTAL}

This investigation was carried out with a Bendix time-of-flight mass spectrometer Model 12-107 which is equipped with a high pressure ion source capable of operating up to $100 \mu \mathrm{Hg}^{2}$ Pressures in the ionization chamber were measured with an MKS Type 77 Baratron pressure meter connected directly to the ionization chamber through a $\frac{3}{8}$-in.-i.d. pipe. Pressures were kept constant during an experiment by using 3 -liter sample gas reservoirs. The gases were introduced into the ion source through two Granville-Phillips variable leaks. In order to obtain fairly stable ionizing electron current, a Lambda power supply, Model LH 121B FM, was used. In all the experiments the electron trap was maintained at $50 \mathrm{~V}$ with respect to the ionization chamber and the ionizing electrons entering the ionization chamber had an energy of about $7 \mathrm{eV}$. This means that the electrons reaching the trap were accelerated to an over-all energy of about $57 \mathrm{eV}$. In order to suppress field penetration into the ionization chamber, a molybdenum mesh of more than $80 \%$ transmission was fitted in the hole in front of the trap.

All the gases used were purchased from Matheson Company, Incorporated and were of $99.6 \%$ purity. They were used without further purification.

Experimental procedures adopted to record the spectra can be briefly described as follows: One of the gases $\mathrm{Ar}, \mathrm{H}_{2}$, or $\mathrm{D}_{2}$ was admitted into the ion source at a pressure of $10 \mu$ and kept constant at that pressure for the entire period of the experiment with the help of the Granville-Phillips needle valve. The other reactant gas was then introduced into the ionization chamber through another needle valve at various pressures and the spectra recorded for each pressure of this second gas from mass 1 to 50. The distance between the trap and the ionization chamber was $4 \mathrm{~mm}$ and that between the trap and the axis passing through the center $P$ (see Fig. 1) of the ionization chamber and the ion exit port was $13 \mathrm{~mm}$.

The ion source was operated in the pulsed mode without applying any delay between the electron beam pulse and the ion focus pulse.

\section{THEORY}

\section{Electron Impact Ionization}

Considering $e_{0}$ as the number of electrons entering the trap region per second and $l_{1}$ the distance between the trap and the ionization chamber, the decay of electron current in the trap region can be represented 


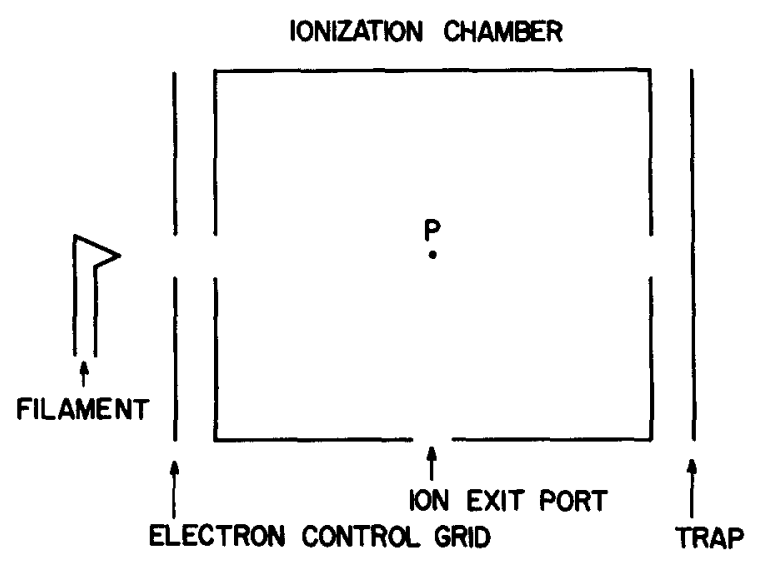

Fig. 1. Schematic diagram of the ion source.

approximately by the relation

$$
e_{f} / e_{0}=\exp \left[-\left(\sigma_{i_{1}} M_{1}+\sigma_{i_{2}} M_{2}\right) l_{1}\right]
$$

where $e_{j}$ is the number of electrons reaching the trap, $\sigma_{i_{1}}$ and $\sigma_{i_{2}}$ the electron impact ionization cross sections of the two gases admitted into the ion source, and $M_{1}$ and $M_{2}$ the concentrations of the corresponding gases. Equation (1) is not rigorously correct. Some ionizing collisions will occur sufficiently far from the anode to permit the ejected electrons to gain energy enough from the field to undergo further ionization. The gases employed, however, have rather small cross sections for ionization. Consequently, even at the highest pressures employed in these experiments the number of ionizing collisions experienced by secondary electrons is much smaller than the number experienced by primary electrons. For this reason we feel that Eq. (1), while not precise, introduces no significant error in the results computed from our data. From Eq. (1) we can write

$$
1-e_{f} / e_{0}=n_{0}{ }^{+} / e_{0}=1-\exp \left[-\left(\sigma_{i_{1}} M_{1}+\sigma_{i_{2}} M_{2}\right) l_{1}\right] \text {, }
$$

where $e_{0}-e_{f}=n_{0}+$ is the number of ions produced immediately before the electrons are collected on the trap. Because ionization cross section increases with electron energy, the greatest number of primary ions will be produced just in front of the trap. Further, these will receive the largest amount of energy in passing out of the trap region and thus will be the first to reach the region of interest immediately above the ion exit port. The peaks tend to show a tailing toward the high mass (longer time) side as would be expected. Because of these facts and to simplify our calculations, we assume that all ions are formed immediately in front of the trap.

For the values of pressure used in this experiment, the exponential term is substantially smaller than unity. Hence, Eq. (2) can be written as

$$
n_{0}{ }^{+} \approx e_{0}\left(\sigma_{i_{1}} M_{1}+\sigma_{i_{2}} M_{2}\right) l_{1} \text {. }
$$

Taking $n_{0_{1}}{ }^{+}$as the number of ions formed in the trap region from the gas of type 1 , then one can write

$$
n_{0_{1}}{ }^{+}=\left[\sigma_{i_{1}} M_{1} /\left(\sigma_{i_{1}} M_{1}+\sigma_{i_{2}} M_{2}\right)\right] n_{0}{ }^{+}
$$

which reduces to

$$
n_{0_{1}}{ }^{+}=n_{0}{ }^{+} e_{0} \sigma_{i_{1}} M_{1} l_{1}
$$

A similar equation, of course, applies to ions of type 2 .

\section{Charge Exchange Ionization}

The ions $n_{0_{1}}{ }^{+}$formed near the trap undergo repulsion by the trap and move towards the ionization chamber. During their passage in this direction, these ions undergo charge exchange and perhaps ion-molecule reactions with both types of neutral species present in the system. If $l_{2}$ is the distance between the trap and the point $P$ (see Fig. 1) just above the ion exit port, and $\sigma_{c 1}$ and $\sigma_{c 2}$ are the charge exchange cross sections for the reaction between this type of ion and the neutral species of type 1 and 2, respectively, then it can be shown that

$$
n_{1}{ }^{+}=n_{0_{1}}{ }^{+} \exp \left[-\left(\sigma_{c_{1}} M_{1}+\sigma_{c_{2}} M_{2}\right) l_{2}\right],
$$

where $n_{1}{ }^{+}$represent the number of ions of type 1 remaining after traversing the distance $l_{2}$. From (5) one can write for $\Delta n_{1}{ }^{+}$, the number of ions of type 1 disappearing by charge exchange directly above the ion exit port,

$$
\begin{gathered}
-\Delta n_{1}{ }^{+} / \Delta l_{2} \approx-d n / d l_{2}=\left(\sigma_{c_{1}} M_{1}+\sigma_{c_{2}} M_{2}\right) n_{1}{ }^{+}, \\
-\Delta n_{1}{ }^{+}=N_{0}{ }^{+}=\Delta l_{2}\left(\sigma_{c_{1}} M_{1}+\sigma_{c_{2}} M_{2}\right) n_{1}{ }^{+},
\end{gathered}
$$

but $\mathrm{N}_{0}{ }^{+}$is the sum of the total number of ions $\mathrm{N}_{0_{1}}{ }^{+}$ and $\mathrm{N}_{\mathrm{O}_{2}}{ }^{+}$formed by charge exchange between the ions of type 1 and the neutral species 1 and 2 above the ion exit port.

Thus,

$$
N_{0_{1}}{ }^{+}=\left[\sigma_{c_{1}} M_{1} /\left(\sigma_{c_{1}} M_{1}+\sigma_{c_{2}} M_{2}\right)\right] N_{0}{ }^{+} .
$$

Combining Eqs. (4) $-(7)$, one gets

$N_{0_{1}}{ }^{+}=n_{0}{ }^{+} e_{0} l_{1} \Delta l_{2} \sigma_{c_{1}} \sigma_{i_{1}} M_{1}^{2} \exp \left[-\left(\sigma_{c_{1}} M_{1}+\sigma_{c_{2}} M_{2}\right) l_{2}\right]$.

Similarly, it can be shown that

$N_{0_{2}}{ }^{+}=n_{0}{ }^{+} e_{0} l_{1} \Delta l_{2} \sigma_{c 2} \sigma_{i_{1}} M_{1} M_{2} \exp \left[-\left(\sigma_{c 1} M_{1}+\sigma_{c 2} M_{2}\right) l_{1}\right]$.

Rearranging and taking logarithms of (8) and (9), one gets

and

$$
\ln \left(N_{0_{1}}{ }^{+} / M_{1}^{2}\right)=A_{1}-\left(\sigma_{c_{1}} M_{1}+\sigma_{c_{2}} M_{2}\right) l_{2}
$$

$$
\ln \left(N_{0_{2}}{ }^{+} / M_{1} M_{2}\right)=A_{2}-\left(\sigma_{c_{1}} M_{1}+\sigma_{c_{2}} M_{2}\right) l_{2},
$$

where $A_{1}=n_{0}{ }^{+} e_{0} l_{1} \Delta l_{2} \sigma_{c_{1}} \sigma_{i_{1}}$ and $A_{2}=n_{0}{ }^{+} e_{0} l_{1} \Delta l_{2} \sigma_{c_{2}} \sigma_{i_{1}}$.

Equation (10) shows that a plot of $\ln \left(N_{0_{1}}{ }^{+} / M_{1}{ }^{2}\right)$ vs $M_{1}$ at constant $M_{2}$ should give a straight line with the slope given by $l_{2} \sigma_{c_{1}}$. Similarly, Eq. (11) also should give a straight line when properly plotted. 


\section{RESULTS AND DISCUSSION}

Considering the $\mathrm{Ar}-\mathrm{H}_{2}$ system, the following four equations can be written for the four major peaks observed in the spectrum:

$$
\begin{aligned}
& \mathrm{H}_{2}+(\rightarrow)+\mathrm{H}_{2} \rightarrow \mathrm{H}_{2}(\rightarrow)+\mathrm{H}_{2}+(\mathrm{I}), \\
& \mathrm{Ar}^{+(\rightarrow)}+\mathrm{H}_{2} \rightarrow \mathrm{Ar}^{(\rightarrow)}+\mathrm{H}_{2}+(\mathrm{II}), \\
& \mathrm{H}_{2}{ }^{+(\rightarrow)}+\mathrm{Ar} \rightarrow \mathrm{H}_{2}(\rightarrow)+\mathrm{Ar}^{+}(\mathrm{I}), \\
& \mathrm{Ar}^{+(\rightarrow)}+\mathrm{Ar} \rightarrow \mathrm{Ar}^{(\rightarrow)}+\mathrm{Ar}^{+}(\mathrm{II}) \text {. }
\end{aligned}
$$

The superscript arrow on any of the ions indicate that these are the primary ions coming from the trap region. The $\mathrm{H}_{2}{ }^{+}$ions of type (I) are formed sooner and arrive at the detector earlier than the $\mathrm{H}_{2}+$ ions of type (II). Similarly, the $\mathrm{Ar}^{+}$ions of type (I) will reach the collector before those of type (II). After charge exchange, the primary ions continue to move ahead, with most of their kinetic energy preserved. Under the conditions employed in this investigation, no $\mathrm{ArH}^{+}$ions and only a faint trace of $\mathrm{H}_{3}{ }^{+}$or $\mathrm{D}_{3}{ }^{+}$ions were observed. These results are explained as follows:

Any primary $\mathrm{Ar}^{+}$ions crossing the reaction zone immediately above the exit port might be expected to pick up a hydrogen or deuterium atom and Giese and Maier ${ }^{11}$ have shown that the cross section for this reaction is small but sufficiently great for $50-\mathrm{V}$ ions for a significant amount of reaction to occur. However, this is a stripping ${ }^{16}$ reaction and the $\mathrm{ArH}^{+}$or $\mathrm{ArD}^{+}$ ions would proceed in the same direction as the original argon ion and so would not be collected. Further, the time required for the primary argon ion to travel from the trap to the reaction zone above the exit port is approximately $1 \mu \mathrm{sec}$ and since no time delay was employed in this study, the ion extraction pulse would be turned on some $0.75 \mu \mathrm{sec}$ before arrival of the argon ion. Thus, any $\mathrm{H}_{2}{ }^{+}$ions formed by charge exchange from primary argon ions would immediately be swept out of the source before they had time to undergo

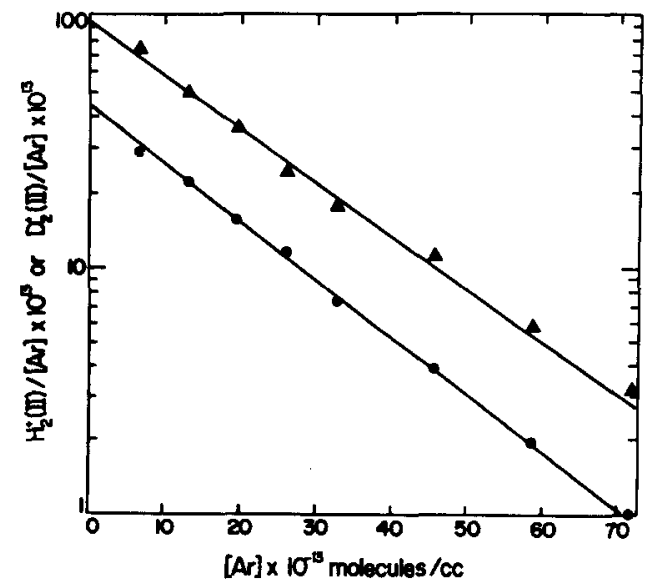

FIG. 2. Variation in intensity of hydrogen ions with added argon at $10 \mu$ hydrogen pressure., $\mathrm{H}_{2} ; \Delta, \mathrm{D}_{2}$.

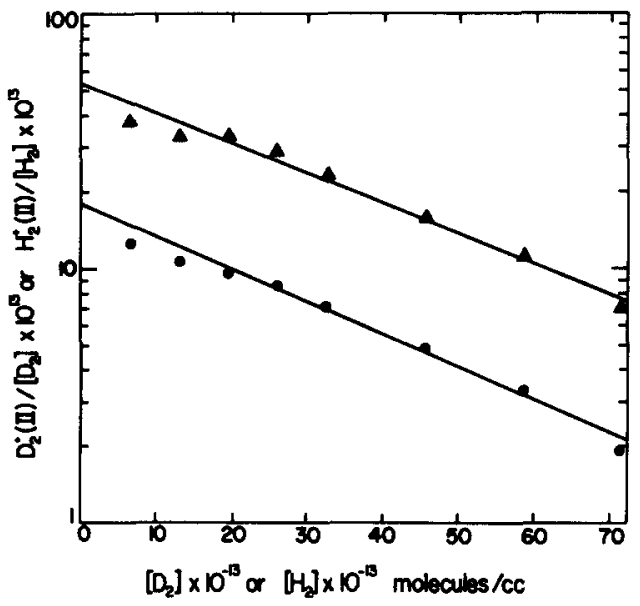

Fra. 3. Variation in intensity of hydrogen ions with added hydrogen at $10 \mu$ argon pressure., $\mathrm{H}_{2} ; \Delta, \mathrm{D}_{2}$.

reaction. Thus, $\mathrm{H}_{3}+$ ions are not observed as arising from charge exchange from $\mathrm{Ar}^{+}$to hydrogen under our conditions.

$\mathrm{H}_{2}{ }^{+}$primary ions traveling from the trap region might be expected to undergo reaction with $\mathrm{H}_{2}$ to give $\mathrm{H}_{3}+$ ions. However, at energies considerably less than $50 \mathrm{~V}$, Giese and Maier ${ }^{11}$ have shown that the cross section for this reaction is vanishingly small so that even if $\mathrm{H}_{3}{ }^{+}$were collectable, it would be formed in amounts too small to be detected. Similarly, $\mathrm{H}_{2}{ }^{+}$or $\mathrm{D}_{2}{ }^{+}$might transfer a proton or deuteron to argon and such ions, if formed, might be expected to be collectable since little or no momentum would be transferred. However, again Giese and Maier ${ }^{11}$ have shown that the cross section for deuteron transfer reaction to argon is very small and similar behavior would be expected of $\mathrm{H}_{2}$. Hence, the ions formed by direct transfer from the primary hydrogen ion beam to argon would be too small to be detected.

The hydrogen ions arrive at the reaction zone at about the same time that the ion extraction pulse is turned on. As a consequence, the secondary hydrogen and argon ions formed by charge exchange from the primary hydrogen ion beam have very little time for reaction befcre they are extracted. We do indeed see a very small amount of $\mathrm{H}_{3}+$ but the intensity is not sufficient to permit quantitative measurements. Probably some $\mathrm{ArH}^{+}$or $\mathrm{ArD}^{+}$was formed by reaction of the secondary hydrogen and argon ions, but the amount would necessarily be quite small because of the very short reaction time. Because of the breadth of the $\mathrm{Ar}^{+}$peak due to the time spread in the primary ion beam, the small $\mathrm{ArH}^{+}$or $\mathrm{ArD}^{+}$ion peaks would be covered by the $\mathrm{Ar}^{+}$ion peak and would not be detected.

It should further be mentioned that as the pressure in the ion source approaches $25 \mu$ and above, the intensities of all of the ion peaks begin to decrease. 
TABLE I. Charge exchange cross sections at 50-V energy.

\begin{tabular}{|c|c|c|c|c|}
\hline & Process & $\begin{array}{c}Q \times 10^{16} \\
\mathrm{~cm}^{2} / \text { molecule } \\
\text { this work }\end{array}$ & $\begin{array}{c}Q \times 10^{16} \\
\mathrm{~cm}^{2} / \text { molecule } \\
\text { work by others }\end{array}$ & Ref. \\
\hline$(1)^{a}$ & $\mathrm{H}_{2}+(\rightarrow)+\mathrm{Ar} \rightarrow \mathrm{H}_{2}(\rightarrow)+\mathrm{Ar}^{+}(\mathrm{I})$ & 32 & & \\
\hline (2) & $\mathrm{H}_{2}+(\rightarrow)+\mathrm{Ar} \rightarrow \mathrm{H}_{2}(\rightarrow)+\mathrm{Ar}^{+}(\mathrm{I})$ & 26 & & \\
\hline (3) & $\mathrm{D}_{2}^{+(\rightarrow)}+\mathrm{Ar} \rightarrow \mathrm{D}_{2}^{(\rightarrow)}+\mathrm{Ar}^{+}(\mathrm{I})$ & 29 & & \\
\hline (4) & $\mathrm{D}_{2}+(\rightarrow)+\mathrm{Ar} \rightarrow \mathrm{D}_{2}(\rightarrow)+\mathrm{Ar}^{+}(\mathrm{I})$ & 26 & & \\
\hline (5) & $\mathrm{Ar}^{+(\rightarrow)}+\mathrm{Ar} \rightarrow \mathrm{Ar}^{(\rightarrow)}+\mathrm{Ar}^{+}(\mathrm{II})^{\mathrm{b}}$ & 41 & 36 & $\mathbf{e}$ \\
\hline (6) & $\mathrm{Ar}^{+(\rightarrow)}+\mathrm{Ar} \rightarrow \mathrm{Ar}^{(\rightarrow)}+\mathrm{Ar}^{+}(\mathrm{II})^{\mathrm{b}}$ & 40 & 35 & f \\
\hline & & & 34 & 10 \\
\hline (7) & $\mathrm{Ar}^{+(\rightarrow)}+\mathrm{Ar} \rightarrow \mathrm{Ar}^{(\rightarrow)}+\mathrm{Ar}^{+}(\mathrm{II})^{0}$ & 40 & 25 & g \\
\hline (8) & $\mathrm{Ar}^{+(\rightarrow)}+\mathrm{Ar} \rightarrow \mathrm{Ar}^{(\rightarrow)}+\mathrm{Ar}^{+}(\mathrm{II})^{\mathrm{c}}$ & 38 & 25 & h \\
\hline (9) & $\mathrm{H}_{2}+(\rightarrow)+\mathrm{H}_{2} \rightarrow \mathrm{H}_{2}(\rightarrow)+\mathrm{H}_{2}+(\mathrm{I})$ & 14 & $8^{d}$ & $\mathrm{i}$ \\
\hline (10) & $\mathrm{D}_{2}+(\rightarrow)+\mathrm{D}_{2} \rightarrow \mathrm{D}_{2}(\rightarrow)+\mathrm{D}_{2}+(\mathrm{I})$ & 13 & 9d & $\mathrm{i}$ \\
\hline (11) & $\mathrm{Ar}^{+(\rightarrow)}+\mathrm{H}_{2} \rightarrow \mathrm{Ar}^{(\rightarrow)}+\mathrm{H}_{2}+(\mathrm{II})$ & 25 & & \\
\hline$(12)$ & $\mathrm{Ar}^{+(\rightarrow)}+\mathrm{H}_{2} \rightarrow \mathrm{Ar}^{(\rightarrow)}+\mathrm{H}_{2}+(\mathrm{II})$ & 22 & & \\
\hline$(13)$ & $\mathrm{Ar}^{+(\rightarrow)}+\mathrm{D}_{2} \rightarrow \mathrm{Ar}^{(\rightarrow)}+\mathrm{D}_{2}+(\mathrm{II})$ & 25 & & \\
\hline (14) & $\mathrm{Ar}^{+(\rightarrow)}+\mathrm{D}_{2} \rightarrow \mathrm{Ar}^{(\rightarrow)}+\mathrm{D}_{2}+(\mathrm{II})$ & 21 & & \\
\hline
\end{tabular}

In processes $(1)-(8), \mathrm{H}_{2}$ or $\mathrm{D}_{2}$ pressure was constant, Ar pressure varied and in processes $9-14, A r$ pressure was constant, $D_{2}$ or $\mathrm{H}_{2}$ pressure varied.

b Processes (5) and (6) in $\mathrm{Ar}-\mathrm{H}_{2}$ system.

${ }^{c}$ Processes (7) and (8) in Ar- $D_{2}$ system.

d Extrapolated from Footnote i.

J. B. Hasted, Proc. Roy. Soc. (London), A205, 421 (1951).

This is probably to be attributed principally to scattering of the slow electrons in the source before they reach the trap region. The resulting loss of intensity, of course, impairs the precision of our measurements at the higher pressures employed.

Some of the experimental results plotted according to Eqs. (10) and (11) are shown in Figs. 2 and 3. In these figures the results obtained for both $\mathrm{Ar}-\mathrm{H}_{2}$ and $\mathrm{Ar}-\mathrm{D}_{2}$ systems are shown.

From these figures it is apparent that the straightline behavior predicted by the Eqs. (10) and (11) fits the experimental data very well. From the slopes of the lines, the values of the charge exchange cross sections for the various processes have been calculated and these are tabulated in Table I. For purposes of comparison, values obtained by other workers by various other methods are also given. In view of the very simple assumptions made in the theoretical calculations above, it is surprising that our values for the cross sections compare favorably with those obtained by others.

It is reasonable to assume that there is negligible transfer of kinetic energy during charge exchange, and as a result the ions formed in this process have translational energies much less than those formed in f J. A. Dillon, W. F. Sheridan, H. D. Edwards, and S, N. Ghosh, J. Chem. Phys. 23, 776 (1955).

R. F. Potter, J. Chem. Phys. 22, 974 (1954).

h H. B. Gilbody and J. B. Hasted, Proc. Roy. Soc. (London) A238, 334 (1957).

i W. H. Cramer, J. Chem. Phys. 35, 836 (1961).

the elastic process. It is quite possible that any of the observed cross sections is an average over the individual values that may arise as a result of the collision between the neutral species and the incident ion beam which may contain ions in various energy states. Vibrationally excited ions might give rise to larger cross sections than those obtained from the ions in their ground state. Slightly larger values of the cross sections found in this investigation as compared to those found by the other workers could be explained, at least in part, if we assume that a considerable number of long-lived excited species reached the ionization chamber from the trap region. Moreover, we must also consider another fact that in the theoretical derivations above we have assumed that the primary ions coming from the trap region travel a distance $l_{2}$ before undergoing detectable charge exchange reactions. In reality, it is quite possible that these ions undergo slight deflection from their original straight-line course while traversing the ionization chamber due to the presence of field gradient inside the chamber. This would then mean that we should take a slightly larger value for $l_{2}$ than that considered in the computations of the cross sections, which would thereby reduce these values to some extent. 


\section{ACKNOWLEDGMENT}

The authors would like to express their appreciation to The Robert A. Welch Foundation for the support of this work.

* Current address: Department of Chemistry, University of Kansas, Lawrence, Kans. 66044.

${ }^{1}$ V. Cermák and Z. Herman, Nucleonics 19, 106 (1961).

2 J. L. Franklin, Yasuo Wada, P. Natalis, and P. M. Hierl, J. Phys. Chem. 70, 2353 (1966).

${ }^{3}$ P. M. Hierl, J. L. Franklin, and D. K. Sen Sharma, Intern. J. Mass Spectrometry Ion Phys. 6, 239 (1971).

${ }^{4}$ F. Wolf, Ann. Physik 28, 361 (1937).

s J. B. Hasted, Proc. Roy. Soc. (London) A212, 235 (1952).

- J. B. H. Stedeford and J. B. Hasted, Proc. Roy. Soc. (London) A227, 466 (1954-1955).
${ }^{7}$ D. P. Stevenson and D. O. Schissler, J. Chem. Phys. 23, 1353 (1955).

${ }^{8}$ H. Gutbier, Z. Naturforsch. 12a, 499 (1957).

${ }^{2}$ D. P. Stevenson and D. O. Schissler, J. Chem. Phys. 29, 282 (1958)

${ }^{10}$ E. Gustafson and E. Lindholm, Arkiv Fysik 18, 219 (1960).

11 C. F. Giese and W. B. Maier, J. Chem. Phys. 39, 739 (1963).

12 M. A. Berta and W. S. Koski, J. Am. Chem. Soc. 86, 5098 (1964).

${ }_{13}$ F. S. Klein and L. Friedman, J. Chem. Phys. 41, 1789 (1964).

${ }_{14}$ T. W. Shannon and A. G. Harrison, J. Chem. Phys. 43, 4206 (1965)

${ }^{15}$ J. B. Homer, R. S. Lehrle, J. C. Robb, and D. W. Thomas, Advan. Mass Spectry. 3, 415 (1966).

${ }^{16} \mathrm{~A}$. Henglein and K. Lackmann, Advan. Mass Spectry. 3, 331 (1966).

THE JOURNAL OF CHEMICAL PHYSICS

VOL UME 56, N UM B E R 3

1 F E B R U A R Y 1972

\title{
Theorem Useful for Interpretation of Magnetic Circular Dichroism Spectra
}

\author{
Llovo Seamans and Albert Moscowitz \\ Department of Chemistry, University of Minnesota, Minneapolis, Minnesota 55455
}

(Received 7 September 1971)

\begin{abstract}
It is shown that the magneto-optical activity associated with a symmetry-forbidden transition is of second or higher order in any perturbation and that the contributions to the magnetic rotational strengths of perturbations belonging to different irreducible representations are additive. This theorem can be useful in analyzing the vibrational structure of a forbidden spectral band and in elucidating the conformations of molecular systems. The problem of the origin dependence of the magnetic rotational strengths is also discussed.
\end{abstract}

\section{INTRODUCTION}

Time-dependent perturbation theory calculations ${ }^{1,2}$ give the following expressions for the contribution of the transition $a \rightarrow j$ to the magneto-optical rotatory dispersion (MORD) and magnetic circular dichroism (MCD) of randomly oriented molecules:

$$
\begin{aligned}
{[\phi(a \rightarrow j)]_{m} } & =-(24 N H / \hbar c)\left(A(a \rightarrow j) f_{1}\left(\nu, \nu_{a j}\right)\right. \\
+ & \left.\{B(a \rightarrow j)+[C(a \rightarrow j) / k T]\} f_{2}\left(\nu, \nu_{j a}\right)\right), \\
{[\theta(a \rightarrow j)]_{m} } & =-(24 N H / \hbar c)\left(A(a \rightarrow j) f_{3}\left(\nu, \nu_{j a}\right)\right. \\
+ & \left.\{B(a \rightarrow j)+[C(a \rightarrow j) / k T]\} f_{4}\left(\nu, \nu_{j a}\right)\right),
\end{aligned}
$$

where $[\theta]_{m}$ and $[\phi]_{m}$ are defined as for natural optical activity, $H$ is the magnetic field strength, and $N$ is Avagadro's number. The magnetic rotational strengths $A, B$, and $C$ are given by ${ }^{3}$

$$
\begin{array}{r}
A(a \rightarrow j)=\left(2 d_{a}\right)^{-1} \sum_{\{a\},\{j\}}(\langle j|\boldsymbol{\mu}| j\rangle-\langle a|\boldsymbol{\mu}| a\rangle) \\
\cdot \operatorname{Im}(\langle a|\mathrm{~m}| j\rangle \times\langle j|\mathbf{m}| a\rangle), \\
B(a \rightarrow j)=\left(d_{a}\right)^{-1} \sum_{\{a\}, \mid j\}} \operatorname{Im}\left[\sum_{k \notin\{a\}}\left(\langle k|\boldsymbol{\mu}| a\rangle / E_{k a}\right)\right. \\
\cdot(\langle a|\mathbf{m}| j\rangle \times\langle j|\mathbf{m}| k\rangle) \\
\left.+\sum_{k \notin\{j\}}\left(\langle j|\boldsymbol{\mu}| k\rangle / E_{k j}\right) \cdot(\langle a|\mathbf{m}| j\rangle \times\langle k|\mathbf{m}| a\rangle)\right], \\
C(a \rightarrow j)=\left(2 d_{a}\right)^{-1} \sum_{\{a),\{j\}}\langle a|\boldsymbol{\mu}| a\rangle \\
\cdot \operatorname{Im}(\langle a|\mathbf{m}| j\rangle \times\langle k|\mathbf{m}| a\rangle),
\end{array}
$$

where $d_{a}$ is the degeneracy of state $a$, the energies $E_{m n}$ are defined as $E_{m}-E_{n}, \mathrm{~m}$ and $\boldsymbol{\mu}$ are the electric and magnetic dipole moment operators, and the sets $\{a\}$ and $\{j\}$ consist of the states degenerate with the states $\Psi_{a}$ and $\Psi_{j}$, respectively. $A(a \rightarrow j)$ is nonzero only if $\Psi_{a}$ or $\Psi_{j}$ is degenerate, and $C(a \rightarrow j)$, the "paramagnetic" term is nonzero only if $\Psi_{a}$ is degenerate. If $\Psi_{a}$ or $\Psi_{j}$ is degenerate, it is required to be diagonal in some component of $\mu$ and hence to be complex.

As can be seen from Eq. (4), a quantitatively accurate theoretical accounting of the observed magnetic rotational strengths requires, in principle, detailed knowledge of the wavefunctions of the states of the system. However, if one is content to use the observed data to provide answers to chemically interesting stereochemical and spectroscopic questions of a qualitative nature, then group theoretical considerations can often obviate the need for detailed calculations, especially in the case of forbidden transitions. It is the purpose of the present paper to elaborate this point of view.

\section{FORBIDDEN TRANSITIONS-SYMMETRY RULES FOR MAGNETO-OPTICAL ACTIVITY}

It is clear from an examination of Eqs. (3)-(5) that the magnetic rotational strengths vanish for an electric dipole forbidden transition. If, upon being subjected to a perturbation, the symmetry of the system is altered in such a way as to make the transition formally allowed, the matrix element $\langle a|\mathbf{m}| j\rangle$ then is of first 